\title{
The effects of dexmedetomidine on biomarkers of oxidative stress and antioxidants in kidney
}

\author{
Akpinar $\mathrm{H}^{1}$, Akpinar $\mathrm{O}^{2}$ \\ Suleyman Demirel University, Unit of Anesthesiology and Reanimation, Department of Oral and Maxillofacial Surgery, \\ Faculty of Dentistry Isparta, Turkey. orhanakpnr@hotmail.com
}

\begin{abstract}
BACKGROUND AND AIM: Cerebral ischemia not only disrupts brain functions, but also damages other organs. We aimed to determine whether two different doses of dexmedetomidine (DEX) had protective effects against the oxidative damage to kidneys.

MATERIALS AND METHODS: Forty-two rats were equally divided into 7 groups. The first group was the control group. Second group members were operated to expose the cerebral artery without inducing cerebral ischemia. The third and fourth groups were DEX treatments groups. The fifth, sixth and seventh group were operated to induce cerebral ischemia. DEX was given to the groups at the 3rd, 24th and 48th hour.

RESULTS: MDA levels in the kidneys were higher in the group with cerebral ischemia. Significant reductions in MDA levels were observed after treatment with DEX in the ISCH group $(p<0.05)$. Decreased GSH-Px activity and reduced glutathione GSH levels in the kidneys were observed with DEX treatments. After treatment, there was a significant increase in $\alpha$-tocopherol and $\beta$-carotene levels $(p<0.01)$.

CONCLUSION: DEX administration during cerebral ischemia had a positive effect on oxidative stress and antioxidants in rat kidney (Tab. 1, Fig. 5, Ref. 31). Text in PDF www.elis.sk.

KEY WORDS: dexmedetomidine, oxidative stress, antioxidants cerebral ischemia, kidney.
\end{abstract}

\section{Introduction}

Cerebral ischemia-induced injury is one of the leading causes of death in the world and leads to a high mortality and disability rate. The long-term outcome for the brain in cerebral ischemiainduced injury includes neuronal diseases (1). Cerebral ischemia not only impairs brain functions, but also leads to disturbances occurring in other organs. Reperfusion injury may occur after the reintroduction of oxygenated blood to ischemic tissues, due to released free oxygen radicals and activated neutrophils (2). It has been proposed that cerebral ischemia is a manifestation of an underlying multi-system endothelial disorder affecting the small vessels of the kidney, brain, heart, and retina (3), possibly mediated through inflammation (4). After acute inflammatory response, secondary organ dysfunction and finally organ failure might occur, due to free oxygen radicals and leukocyte aggregation (5-7). After the cerebral ischemic injury, the generation of reactive oxygen species (ROS) may contribute to the neurodegenerative dis-

${ }^{1}$ Suleyman Demirel University, Unit of Anesthesiology and Reanimation, Department of Oral and Maxillofacial Surgery, Faculty of Dentistry Isparta, Turkey, and ${ }^{2}$ Suleyman Demirel University, Instutute of Health Sciences, Medical Microbiology Department Isparta, Turkey

Address for correspondence: O. Akpinar, Suleyman Demirel University, Institute of Health Sciences. Dogu kampusu Cunur, 32260 Isparta, Turkey. Phone: +5055788879

Acknowledgements: Analyses of the study was performed by the Neuroscience Research Centre of SDU. ease process through alterations in the structure of DNA, RNA, proteins and lipids $(8,9)$. The imbalance between the production of ROS and their elimination by protective mechanisms leads to oxidative stress. This response occurs in various pathophysiological conditions such as: aging, inflammation, cardiovascular and neurodegenerative diseases, damaging many components including proteins, DNA/RNA and lipids $(10,11)$. Several agents were used in animal studies to protect organs from ischemia induced oxidative stress. An approved agent for the indicated clinical use with protective effects against ischemia-reperfusion injury of the kidney is dexmedetomidine, a selective and potent $\alpha 2$-adrenoceptor agonist. It is frequently used for anesthesia in daily practice and for sedation, anxiolysis, and analgesia in the intensive care unit (12-15). In this experimental study, we aimed to determine whether two different doses of dexmedetomidine had protective effects against the oxidative damage to kidneys in the cerebral ischemia induction model on rats.

\section{Materials and methods}

Animals

All animal procedures and protocols were performed in accordance with The Guide for the Care and Use of Laboratory Animals and were reviewed and approved by the local experimental animal ethical committee of Suleyman Demirel University (Protocol number: 09-07-15/04). Alldata about the animals and induction of cerebral ischemia methods were given in our previous study (15). 
Tab. 1. The effects of DEX4 (4 g/kg) and DEX40 (40 g/kg) treatments on lipid peroxidation (MDA), reduced glutathione (GSH), antioxidant vitamin concentrations and glutathione peroxidase (GSH-Px) activity in kidney of cerebral ischemia (ISCH)-induced rats ( $($ mean \pm SD and $\mathbf{n}=6$ ).

\begin{tabular}{|c|c|c|c|c|c|c|c|}
\hline Parameters & Control & Sham & DEX4 & DEX40 & ISCH & $\mathrm{ISCH}+\mathrm{DEX} 4$ & $\mathrm{SCH}+\mathrm{DEX} 40$ \\
\hline $\begin{array}{l}\text { MDA } \\
(\mu \mathrm{mol} / \mathrm{g} \text { protein })\end{array}$ & $19.50 \pm 0.97$ & $19.50 \pm 1.76$ & $18.40 \pm 1.95$ & $16.90 \pm 1.32$ & $20.30 \pm 1.69^{\mathrm{d}}$ & $18.10 \pm 1.77^{\circ}$ & $17.30 \pm 1.65^{\mathrm{c}}$ \\
\hline $\begin{array}{l}\mathrm{GSH} \\
(\mu \mathrm{mol} / \mathrm{g} \text { protein })\end{array}$ & $10.10 \pm 0.44$ & $9.45 \pm 0.51$ & $10.40 \pm 0.81$ & $10.70 \pm 0.80$ & $8.94 \pm 0.61^{\mathrm{a}}$ & $10.10 \pm 0.77^{\mathrm{c}}$ & $10.20 \pm 0.53^{c}$ \\
\hline $\begin{array}{l}\text { GSH-Px } \\
\text { (IU/g protein) }\end{array}$ & $23.30 \pm 1.14$ & $23.40 \pm 3.22$ & $24.10 \pm 2.59$ & $23.00 \pm 1.52$ & $22.20 \pm 1.95$ & $24.90 \pm 3.38$ & $24.60 \pm 1.61$ \\
\hline $\begin{array}{l}\beta \text {-carotene } \\
(\mu \mathrm{mol} / \mathrm{g} \text { kidney })\end{array}$ & $0.81 \pm 0.10$ & $0.75 \pm 0.14$ & $0.67 \pm 0.11$ & $0.69 \pm 0.04$ & $0.82 \pm 0.12^{\mathrm{a}}$ & $0.86 \pm 0.11^{\mathrm{d}}$ & $0.86 \pm 0.13^{\mathrm{d}}$ \\
\hline $\begin{array}{l}\alpha \text {-tocopherol } \\
(\mu \mathrm{mol} / \mathrm{g} \text { kidney })\end{array}$ & $11.70 \pm 2.08$ & $11.20 \pm 1.00$ & $12.40 \pm 0.81$ & $12.10 \pm 1.13$ & $10.10 \pm 1.19^{\mathrm{b}}$ & $12.10 \pm 1.77^{\mathrm{d}}$ & $12.30 \pm 0.51^{\mathrm{d}}$ \\
\hline
\end{tabular}

${ }^{\mathrm{a}} \mathrm{p}<0.05$ and ${ }^{\mathrm{b}} \mathrm{p}<0.01$ versus control, sham, DEX4 and DEX40 groups, ${ }^{\mathrm{c}} \mathrm{p}<0.05$ and ${ }^{\mathrm{d}} \mathrm{p}<0.01$ versus ISCH group

\section{Experimental design}

The rats were equally divided into seven groups $(n=6)$, as followed:

Control Group: Non-ischemic, non-supplemented rats receiving physiologic saline solution $(0.9 \% \mathrm{w} / \mathrm{v})$ via intraperitoneal injection (IP) at the 3rd, 24th and48th hour.

Sham Group: The group was operated to expose the cerebral artery, and the incision was then closed without inducing cerebral ischemia. The rats in this group received physiologic saline solution via IP injection at the 3rd, 24th and 48th hour after the operation.

DEX4 Group: Non-ischemic rats treated with Dexmedetomidine (Precedex flk, Meditera Group, Turkey) 4 g/kg, via IP injection at the 3rd, 24th and 48th hour.

DEX40 Group: Non-ischemic rats treated with Dexmedetomidine $40 \mathrm{~g} / \mathrm{kg}$ via IP injection at the $3 \mathrm{rd}, 24$ th and 48th hour.

Ischemic Group (ISCH): Cerebral ischemia was induced by 30 min of cerebral artery occlusion, then IP injection of physiologic saline solution was applied at the 3rd, 24th and 48th hours.

Ischemic + DEX4 Group (ISCH+DEX4): Ischemic rats, treated with DEX $4 \mathrm{~g} / \mathrm{kg}$ via IP injection at the 3rd, 24th and 48th hour after cerebral ischemia induction.

Ischemic + DEX40 Group(ISCH+DEX40): Ischemic rats, treated with DEX $40 \mathrm{~g} / \mathrm{kg}$ via IP injection at the $3 \mathrm{rd}$, 24th and 48th hour after cerebral ischemia induction.

Twelve hours after the last DEX and physiological saline administration, all rats were sacrificed via cervical dislocation and kidney samples were taken.

\section{Preparation of kidney tissue samples}

Kidneys were stored in a deep freeze $\left(-85^{\circ} \mathrm{C}\right)$ until processing. kidney sample was placed on ice, and homogenized by an ultrasonic homogenization (SONOPULS HD 2070, Bandelin Electronic, Berlin,Germany). All preparation procedures were performed on ice. The homogenates were analyzed for estimation of tissue MDA, GSH levels, GPx activity and antioxidant vitamin levels.

\section{Analyses of the kidney samples homogenates}

Analyses of the study were performed by the Neuroscience Research Centre (NÖROBAM) of SDU, after approval of the
NÖROBAM scientific committee (Protocol no: 13.06.2015-02$01)$. The MDA levels were measured by the method of Placer et al (16). The results were expressed as $\mu \mathrm{mol} / \mathrm{g}$ protein in the kidney samples. Tissue protein determination was achieved by the method described by Lowry et al (17). The GSH contents in the kidney samples were spectrophotometrically (UV1800, SHIMADZU, Kyoto, Japan) measured by the method of Sedlak et al (18). GSHPx activities of the kidney were measured spectrophotometrically according to method of the Lawrence at al (19). GSH-Px activity and GSH level in the kidney were expressed as IU/g protein and $\mu \mathrm{mol} / \mathrm{g}$ protein, respectively. Vitamin E ( $\alpha$-tocopherol) concentrations were determined in the kidney samples by a modified method of Desai (20). $\beta$-carotene concentrations in the tissue samples were analyzed by a modified method of Suzuki and Katoh (21).

\section{Statistical analysis}

The results are presented as the mean \pm standard deviation .The SPSS Statistical program (17.0, SPSS Inc. Chicago, Illinois, USA) was used for the statistical analysis of the data. The Mann-Whitney U test was used to establish the significance of differences among the four groups. The significance level was set at $\mathrm{p}<0.05$.

\section{Results}

\section{Malonyldialdehyde values}

The results showed that MDA levels in the kidney samples in the cerebral ischemia group were significantly $(p<0.01)$ higher than in the control, sham, DEX4 and DEX40 groups. Therefore, the oxidative stress level in the kidney of the rats was higher because of the induction of cerebral ischemia. However, administrations of DEX4 and DEX40 caused decrease in the MDA level of the rat's kidney samples. In other words, the MDA levels in the kidney samples were lower in the DEX4 and DEX40 groups as compared to the control and the sham control groups $(\mathrm{p}<$ $0.05)$. The MDA levels were higher in the ischemia group $(\mathrm{p}<$ 0.05 ) compared to the control, sham, DEX4 and DEX40 groups. However, the MDA levels in the ischemia + DEX4 and ischemia + DEX40groups were significantly $(p<0.05)$ lower than in the ischemia group (Fig. 1). 
$476-480$

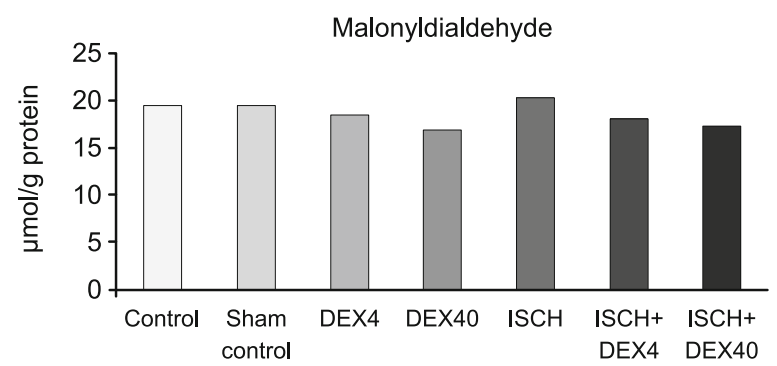

Fig. 1. The effects of DEX4 $(4 \mathrm{~g} / \mathrm{kg})$ and DEX40 $(40 \mathrm{~g} / \mathrm{kg})$ treatments on MDA level kidney of cerebral ischemia (ISCH)-induced rats (mean $\pm S D$ and $n=6$ ).

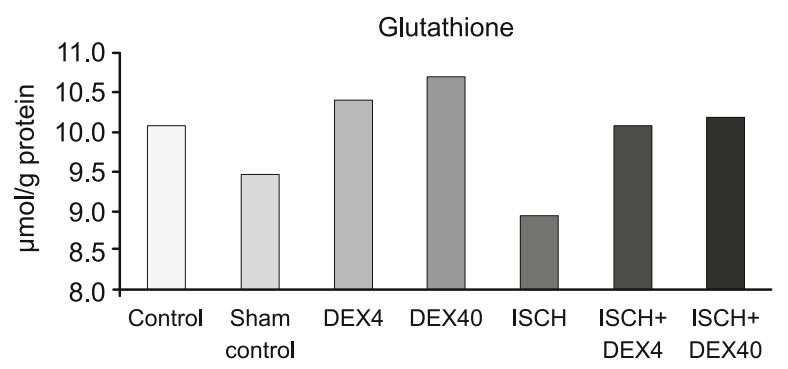

Fig. 2. The effects of DEX4 (4 g/kg) and DEX40 (40 g/kg) treatments on GSH level kidney of cerebral ischemia(ISCH)-induced rats (mean \pm SD and $n=6$ ).

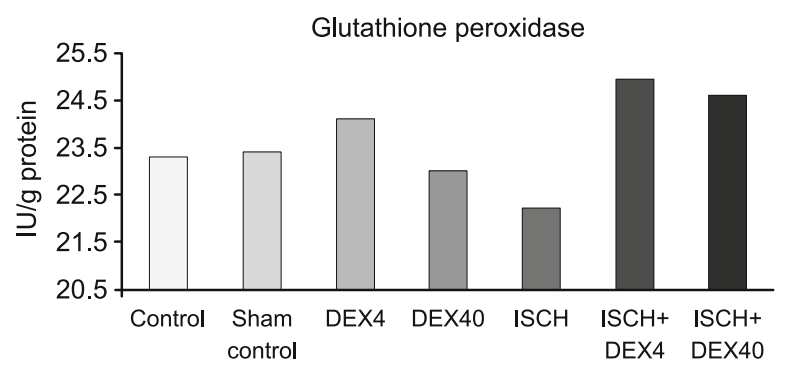

Fig. 3. The effects of DEX4 (4 g/kg) and DEX40 (40 g/kg) treatments on GSH-Px activity kidney of cerebral ischemia(ISCH)-induced rats (mean \pm SD and $n=6$ ).

\section{GSH-Px activity and GSH level}

GSH concentration in the kidney samples $(\mathrm{p}<0.05)$, and GSH-PX activities in the kidney samples ( $p<0.01)$, were significantly lower in the cerebral ischemia group compared to the control and sham control groups. The kidney GSH levels were also higher in the DEX4 and DEX40 groups compared to the control and sham control groups $(\mathrm{p}<0.05)$. However, GSH concentration $(\mathrm{p}<0.05)$ and GSH-Px activity in the kidney $(\mathrm{p}<0.01)$ were markedly increased by the two (DEX4 and DEX40) treatments (Figs 2 and 3).

\section{Antioxidant vitamin concentrations}

$\beta$-carotene $(\mathrm{p}<0.01)$ and $\alpha$-tocopherol $(\mathrm{p}<0.01)$ concentrations in the kidney samples were significantly higher in the DEX4, DEX40 and ischemia groups compared to the control and sham

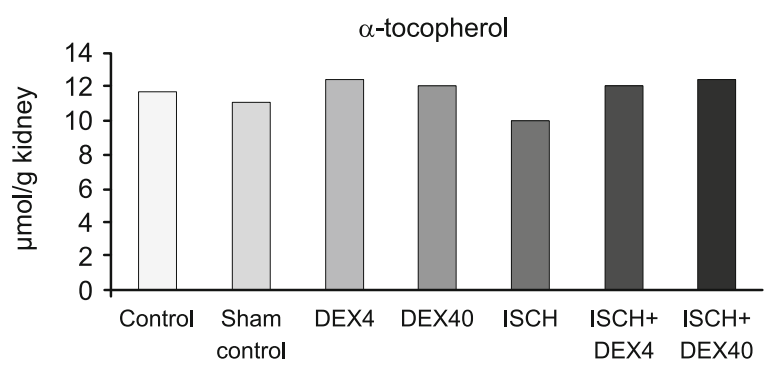

Fig. 4. The effects of DEX4 (4 g/kg) and DEX40 (40 g/kg) treatments on $\alpha$-tocopherol level kidney of cerebral ischemia(ISCH)-induced rats (mean \pm SD and $n=6$ ).

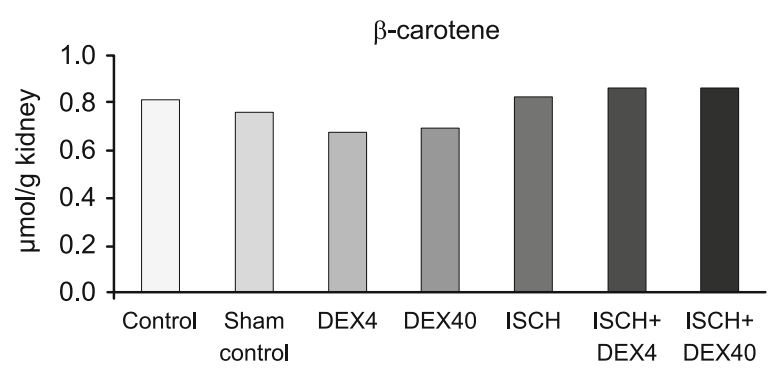

Fig. 5. The effects of DEX4 (4 g/kg) and DEX40 $(40 \mathrm{~g} / \mathrm{kg})$ treatments on $\beta$-carotene level kidney of cerebral ischemia (ISCH)-induced rats $($ mean $\pm S D$ and $n=6)$.

control groups. $\beta$-carotene, and $\alpha$-tocopherol concentrations were improved by the DEX4 and DEX40 administration $(\mathrm{p}<0.001)$. The kidney antioxidant vitamin concentrations were higher in the $\mathrm{ISCH}+\mathrm{DEX} 4$ and ISCH + DEX40 groups compared to the ISCH group $(\mathrm{p}<0.05)$ (Figs 4 and 5).

\section{Discussion}

In the current study it has been observed that there was an increase in the levels of MDA and a decrease in the levels of GSH and GPx levels in the kidney tissue of the rats as the results of the cerebral ischemia. These findings suggest that oxidative stress response after cerebral iscemia plays an important role in the remote organ injury. Magdi et al (22) reported that the ischemiareperfusion injury triggered a systemic inflammatory response and multiple organ dysfunction due to free oxygen radicals and leukocyte aggregation. Many defence systems were developed in the body to prevent cellular damage of free radicals. These are called antioxidant defence systems. Antioxidants prevent lipid peroxidation and cell damage by inhibiting peroxidation chain reactions or by eliminating free radicals. The main endogenous antioxidants include superoxide dismutase, glutathione peroxidase, catalase, $\beta$-carotene, $\alpha$-tocopherol, ascorbic acid, glutathione, ceruloplasmin, transferrin and ferritin (29). The imbalance between the production of reactive oxygen (ROS) species and elimination by protective mechanisms leads to oxidative stress. Oxidative stress is highly correlated with a wide variety of inflammatory and metabolic disease states, including ischaemia (22-24). 
Ischemic injuries contribute to increased ROS production and these are important problems that all the tissues are faced with in the presence of inadequate tissue oxygenation. The free radicals attack cell membrane, protein, DNA or RNA, and extracellular matrix and change their structure and then cause multiple organ dysfunctions involving kidney damage. Oxidative damage induced by reactive oxygen species (ROS) plays an important role in the pathogenesis of acute kidney injury $(15,25-27)$. Free oxygen radicals lead to membrane lipid peroxidation and the final product of this reaction is malondialdehyde (28). Oxidative damage is aggravated by a decrease in antioxidant enzyme activities such as: glutathione and glutathione peroxidase, which acts as a free radical scavengers in conditions associated with oxidative stress (29). GSH can help maintain normal immune system function, and has a function in antioxidation and integrated detoxification. Therefore, GSH plays an important role in protecting cells against oxidative injury (30). GSH-Px is a powerful reducing agent and can eliminate the damage caused by free radicals in the cells. Dexmedetomidine, a highly selective and potent $\alpha 2$ adrenoceptor agonist, has sedative, analgesic, sympatholytic, and hemodynamic stabilizing properties and has been widely investigated in a variety of ischemic models and it was shown to have protective effect on brain, lung, intestine, liver, cardiac, testicular, and kidney tissues in animal models (12-15). Dex is already in clinical use as a sedative for intensive care unit patients, who require only mild sedation and it seems to enhance renal function also in some clinical cases $(31,22)$.

In the current study it has been also observed that there was an increase in the levels of MDA and a decrease in the levels of GSH, and GPx levels in the kidney tissue of rats. These findings suggest that oxidative stress response after cerebral ischemia plays an important role in the remote organ injury. Increase of oxidative stress and deterioration of systemic reactions cause a remote organ dysfunction caused by cerebral ischaemia. Cerebral injury leads to oxidative stress or damage in remote organs, by causing a decrease in the activities of antioxidant enzymes. On contrast, DEX treatment significantly decreases MDA and increases GSH, GPx levels and antioxidant vitamin concentrations in the kidney of cerebral ischemia-induced rats. This indicates that DEX can alleviate renal oxidative injury in the kidney with cerebral ischemia-induced rats. The increased antioxidant vitamin concentrations in the kidney were improved by the DEX4 and DEX40 administration. However, the treatment induced an oxidative toxicity in the kidney.

Kocoglu et al. reported that dexmedetomidine can decrease ischemic injury on kidneys and may increase tolerance to renal injury under ischemic conditions (27).

\section{Conclusion}

To the best of our knowledge, the current study is the first to compare the drug DEX with particular reference to its effects on oxidative stress, and the antioxidant redox system in experimental cerebral ischemia-induced oxidative injury in rats. By reducing the inflammatory response, DEX was able to reduce a remote kidney injury. We found out that the $4 \mathrm{mg} / \mathrm{kg}$ and $4 \mathrm{mg} / \mathrm{kg}$ doses of DEX had a protective effect on the oxidative stress occured during cerebral ischaemia in the kidney. The progression of oxidative injuries of the kidney induced by cerebral ischemia might be modulated by dexmedetomidine as a potent antioxidant drug. However, there is a need of further studies investigating the clinical use of DEX for ischemic damages of the kidney.

\section{References}

1. Lynch JK, Nelson KB. Epidemiology of perinatal stroke. Curr Opin. Pediatr 2001; 13 (6): 499-505.

2. Grace PA. Ischaemia-reperfusion injury. Br J Surg 1994; 81 (5): 637647

3. Thompson CS, Hakim AM. Living beyond our physiological means: small vessel disease of the brain is an expression of a systemic failure in arteriolar function: a unifying hypothesis. Stroke 2009; 40 (5): 322-330

4. Lavallee PC, Labreuche J, Faille D, Huisse MG, Nicaise-Roland P, Dehoux M, Klein IF. Circulating markers of endothelial dysfunction and platelet activation in patients with severe symptomatic cerebral small vessel disease. Cerebrovasc Dis 2013; 36 (2): 131-138.

5. Garbaisz D, Turóczi Z, Fülöp A, Rosero O, Arányi P, Ónody P, Szijártó A. Postconditioning can reduce long-term lung injury after lower limb ischemia-reperfusion. Magy Seb 2013; 66 (3): 146-154.

6. Chen LN, Yang XH, Nissen DH, Chen YY, Wang LJ, Wang JH, Zhang LY. Dysregulated renin-angiotensin system contributes to acute lung injury caused by hind-limb ischemia-reperfusion in mice. Shock 2013; 40 (5): 420-429.

7. Yun X, Maximov VD, Yu J, Vertegel AA, Kindy M. SNanoparticles for targeted delivery of antioxidant enzymes to the brain after cerebral ischemia and reperfusion injury. J Cereb Blood Flow Metab 2013; 33 (4): 583-592.

8. Schieber M, Chandel, N.S. ROS function in redox signaling and oxidative stress. Curr Biol 2014; 24 (10): 453-462.

9. Boccatonda A, Tripaldi R, Davi G, Santilli F. Oxidative StressModulation Through Habitual Physical Activity. Curr Pharm Des 2016; 22 (24): 3648-3680.

10. Islam MT. Oxidative stress and mitochondrial dysfunction-linked neurodegenerative disorders. Neurol Res 2017; 39 (1): 73-82.

11. Rani V, Deep G, Singh RK. Oxidative stress and metabolic disorders: Pathogenesis and therapeutic strategies. Life Sci 2016; 148: 183-193.

12. Lempiainen J, Finckenberg P, Mervaala EE, Storvik M, Kaivola J, Lindstedt K, Mervaala EM. Dexmedetomidine preconditioning ameliorates kidney ischemia-reperfusion injury. Pharmacol Res Perspect 2014; 2 (3): 245.

13. Maier C, Steinberg GK, Sun GH, Zhi GT, Maze M. Neuroprotection by the alpha 2-adrenoreceptor agonist dexmedetomidine in a focal model of cerebral ischemia. Anesthesiology 1993; 79 (2): 306-312.

14. Gu J, Sun P, Zhao H, Watts HR, Sanders RD, Terrando N, Ma D. Dexmedetomidine provides renoprotection against ischemia-reperfusion injury in mice. Crit Care 2011; 15 (3): 153.

15. Akpinar O, Naziroğlu M, Akpinar H. Different doses of dexmedetomidine reduce plasma cytokine production, brain oxidative injury, PARP and caspase expression levels but increase liver oxidative toxicity in cerebral ischemia-induced rats. Brain Res Bull 2017; 130: 1-9. 
476-480

16. Placer ZA, Cushman L, Johnson BC. Estimation of products of lipidperoxidation (malonyl dialdehyde) in biological fluids. Anal Biochem 1966; 16: 359-364.

17. Lowry OH, Rosebrough NJ, Farr AL. Protein measurementwith the Folin-Phenol reagent. J Biol Chem 1951; 193: 265-275.

18. Sedlak J, Lindsay RHC. Estimation of total, protein bound and nonproteinsulfhydryl groups in tissue with Ellmann's reagent. Anal. Biochem 1968; 25: 192-120.

19. Lawrence RA, Burk RF. Glutathione peroxidase activity inseleniumdeficient rat liver. Biochem. Biophys. Res Commun 1976; 71 (4): 952-958.

20. Desai ID. Vitamin E analysis methods for animal tissues. Methods Enzymol 1984; 105:138-147

21. Suzuki J, Katoh N. A simple and cheap method for measuring serum vitamin A in cattle using only a spectrophotometer. Jpn J Vet Sci 1990; 52 (6): 1281-1283.

22. Magdi M, Yassin I, Harkin DW, Halliday MI, Rowlands BJ. Lower Limb Ischemia-Reperfusion Injury Triggers a Systemic Inflammatory Response and Multiple Organ Dysfunction. World J Surg 2002; 26 (1): $115-121$.

23. Sinclair AJ, Barnett AH, Junec J. Free Radicals and Antioxidant Systems in Health and Disease, British J. Hospital Medicine 1990; 43 (5): 334-344.

24. Sonta T, Inoguchi T, Tsubouchi H, Sekiguchi N, Kobayashi K, Matsumoto S, Nawata H. Evidence for contribution of vascular NAD (P)H oxidase to increased oxidative stress in animal models of diabetes andobesity. Free Radic Biol Med 2004; 37 (1): 115-115
25. Frumento RJ, Logginidou HG, Wahlander S, Wagener G, Playford HR, Sladen RN. Dexmedetomidine infusion is associated with enhanced renal function after thoracic surgery. J Clin Anesth 2006; 18 : $422-426$.

26. Magdi M, Yassin I, Harkin DW, Halliday MI, Rowlands BJ. Lower Limb Ischemia-Reperfusion Injury Triggers a Systemic Inflammatory Response and Multiple Organ Dysfunction. World J Surg 2002; 26 (1): $115-121$.

27. Kocoglu H, Ozturk H, Ozturk H, Yilmaz F, Gulcu N. Effect of dexmedetomidine on ischemia-reperfusion injury in rat kidney: a histopathologic study. Ren Fail 2009; 31 (1): 70-74

28. Furukawa S, Fujita T, Shimabukuro M, Iwaki M, Yamada Y, Nakajima Y, Shimomura I. Increased oxidative stress in obesity and its impact on metabolic syndrome. J Clin Investig 2004; 114 (12): 1752-1761.

29. Neeti Sharma. Free Radicals, Antioxidants and Disease. Biol Med 2014; 6 (3): 1 .

30. Blokhina O, Virolainen E, Fagerstedt KV. Antioxidants, Oxidative Damage and Oxygen Deprivation Stress a Review. Ann Bot 2002; 91 (2): 179-179.

31. Frumento RJ, Logginidou HG, Wahlander S, Wagener G, Playford HR, Sladen RN. Dexmedetomidine infusion is associated with enhanced renal function after thoracic surgery. J Clin Anesth 2006; 18 : $422-426$. 